\title{
A New Class of Symmetric Beta Type Distributions Constructed by Means of Symmetric Bernstein Type Basis Functions
}

\author{
Fusun Yalcin *D and Yilmaz Simsek \\ Department of Mathematics, Faculty of Science University of Akdeniz, TR-07058 Antalya, Turkey; \\ ysimsek@akdeniz.edu.tr \\ * Correspondence: fusunyalcin@akdeniz.edu.tr
}

Received: 14 April 2020;

Corrected: 30 January 2022

Accepted: 6 May 2020; $\quad$ Published: 7 May 2020;

\begin{abstract}
The main aim of this paper is to define and investigate a new class of symmetric beta type distributions with the help of the symmetric Bernstein-type basis functions. We give symmetry property of these distributions and the Bernstein-type basis functions. Using the Bernstein-type basis functions and binomial series, we give some series and integral representations including moment generating function for these distributions. Using generating functions and their functional equations, we also give many new identities related to the moments, the polygamma function, the digamma function, the harmonic numbers, the Stirling numbers, generalized harmonic numbers, the Lah numbers, the Bernstein-type basis functions, the array polynomials, and the Apostol-Bernoulli polynomials. Moreover, some numerical values of the expected values for the logarithm of random variable are given.
\end{abstract}

Keywords: Bernstein basis functions; moment generating function; Stirling numbers; digamma function; symmetric beta distributions; Apostol-Bernoulli polynomials; symmetry property random variable; harmonic numbers; Lah numbers

MSC: 05A15; 11B68; 11B73; 11B83; 11S80; 26C05; 42A61; 60E05; 60E10; 62E15

\section{Introduction}

Symmetric probability distribution, symmetric random variables, and the symmetric product of two independent random variables have many applications in pure and applied mathematics. The symmetric beta distribution has also very important applications in scientific studies. Using the beta distribution, some mathematical models related to the behavior of random variables, limited to intervals of finite length have been studied. For this reason, this distribution and its applications have been used in mathematics, in probability, in theoretical statistics, in science, in health science, and in social sciences (cf. [1-5] and the references cited therein). For instance, Gupta and Nadarajah [2] studied the centrality of beta distributions in Bayesian inference with the help of the beta-binomial model. In order to detect defects in a gear, Oguamanam et al. [4] gave the kurtosis of the beta distribution model. On the other hand, generating functions for special numbers and polynomials have been used in the mentioned above areas (cf. [1-4,6-38]).

In this paper, we use the generating function with their functional equations method. By using this method, many identities and relations of the Bernstein-type basis functions are found. By using the Bernstein-type basis functions, we defined beta type distributions. By applying gamma function, beta function, and integral technique, some properties of beta type distributions are given. Using generating functions with their functional equations technique and integral technique, 
many identities and relations involving beta type distributions, the Bernstein-type basis functions, moment generating functions, expected values for the logarithm of random variable, the Stirling numbers, the Apostol-Bernoulli numbers, and the digamma function are given.

Expected values for the logarithm of random variable is very important tool in order to evaluate of the value for the logarithm of the geometric mean of a distribution. As for the expected value of the beta distribution, its computation is based on integral techniques. Furthermore, we give further remarks and observations about the results of this paper.

Throughout this paper, we use the following notations, definitions, and relations.

Let $\mathbb{N}, \mathbb{Z}, \mathbb{Q}, \mathbb{R}$, and $\mathbb{C}$ denote the set of natural numbers, the set of integers, the set of rational numbers, the set of real numbers, and the set of complex numbers, respectively.

$$
\mathbb{N}_{0}=\mathbb{N} \cup\{0\} .
$$

$$
x_{(k)}=x(x-1)(x-2) \ldots(x-k+1)
$$

and

$$
x_{(0)}=1
$$

where $k \in \mathbb{N}_{0}$ (cf. [38]).

In this paper, we give numerical values of the expected value for the logarithm of random variable of the beta type distribution. These values are given in terms of the polygamma function, the digamma function. Therefore, we give a brief description of these function as follows.

The polygamma function of order $n$, which is a meromorphic function on the complex plane, is defined as follows,

$$
\psi^{(n)}(z)=\frac{d^{n+1}}{d z^{n+1}}\{\ln (\Gamma(z))\},
$$

where $\Gamma(z)$ denotes the gamma function, which is the Mellin transform of the negative exponential function $e^{-t}$, defined by

$$
\Gamma(z)=\int_{0}^{\infty} t^{z-1} e^{-t} d t
$$

where $z=x+i y \in \mathbb{C}$ and $x, y \in \mathbb{R}$ with $x>0$ (cf. $[3,24,37,38]$ ).

Observe that the polygamma function is the $(n+1)$ th derivative of the function $\ln (\Gamma(z))$. For $n=0$, the polygamma function reduces to the digamma function $\psi(z)$, which is given by

$$
\psi(z)=\frac{d}{d z}\{\ln (\Gamma(z))\}=\frac{1}{\Gamma(z)} \frac{d}{d z}\{\Gamma(z)\}
$$

(cf. $[3,24,37,38]$ and the references cited therein).

\subsection{Generating Functions for Special Numbers and Polynomials}

In order to give identities, relations, and formulas, including the Apostol-Bernoulli polynomials, the Stirling numbers, the aray polynomials, and Bernstein-type basis functions, we need the following generating functions.

The Apostol-Bernoulli polynomials $\mathcal{B}_{n}^{(m)}(x ; \lambda)$ are defined by the following generating function,

$$
F_{B}(t, x, m ; \lambda)=\left(\frac{t}{\lambda e^{t}-1}\right)^{m} e^{t x}=\sum_{n=0}^{\infty} \mathcal{B}_{n}^{(m)}(x ; \lambda) \frac{t^{n}}{n !}
$$

where $m \in \mathbb{N}$ and $\lambda \in \mathbb{C}$.

Substituting $x=0$ into (1), we have the Apostol-Bernoulli $\mathcal{B}_{n}^{(m)}(\lambda)=\mathcal{B}_{n}^{(m)}(0 ; \lambda)$ (cf. $[22,33,37,38]$ and the references cited therein). 
The $\lambda$-array polynomials $S_{k}^{n}(x ; \lambda)$ are defined by the following generating function,

$$
F_{A}(t, x, k ; \lambda)=\frac{\left(\lambda e^{t}-1\right)^{k}}{k !} e^{t x}=\sum_{n=0}^{\infty} S_{k}^{n}(x ; \lambda) \frac{t^{n}}{n !},
$$

where $k \in \mathbb{N}_{0}$ and $\lambda \in \mathbb{C}$ (cf. [33]).

Substituting $x=0$ into (2), we have the $\lambda$-Stirling numbers $S_{2}(n, k ; \lambda)$, which are given by

$$
F_{S}(t, k ; \lambda)=\frac{\left(\lambda e^{t}-1\right)^{k}}{k !}=\sum_{n=0}^{\infty} S_{2}(n, k ; \lambda) \frac{t^{n}}{n !}
$$

(cf. $[21,33,36-38])$.

Substituting $\lambda=1$ into (3), we have the Stirling numbers of the second kind:

$$
S_{2}(n, k)=S_{2}(n, k ; 1) .
$$

These numbers are also given by the following relation,

$$
x^{n}=\sum_{k=0}^{n} S_{2}(n, k) x_{(k)}
$$

where $k, n \in \mathbb{N}_{0}$ (cf. $[9,21,25,33,36,38] ;$ and the references cited therein).

The Stirling numbers of the first kind $S_{1}(n, k)$ are defined the following generating functions,

$$
F_{S 1}(t, k)=\frac{(\log (1+t))^{k}}{k !}=\sum_{n=0}^{\infty} S_{1}(n, k) \frac{t^{n}}{n !},
$$

and

$$
x_{(n)}=\sum_{k=0}^{n} S_{1}(n, k) x^{k},
$$

where $k, n \in \mathbb{N}_{0}$, and $S_{1}(n, k)=0$ if $k>n$ (cf. $[9,21,25,33,36,38]$ and the references cited therein).

The definition (7) provides us with a generalization and unification of the Bernstein basis functions, which were considered in many earlier investigations by (among others) Simsek [28-35], Farouki [11], Goldman [12], Kim [15], Bayad et al. [7], Li and Goldman [18], and Acikgoz and Araci [6].

Definition 1. ([34]) Let $a$ and $b$ be non-negative real parameters with $a \neq b$. Let $t \in \mathbb{C}$. Let $m$ be a positive integer and let $x \in[a, b]$. The Bernstein-type basis functions of degree $n, \mathbb{Y}_{k}^{n}(x ; a, b, m)$, are defined by

$$
f_{\mathbb{Y}, k}(x, t ; a, b, m)=\frac{t^{k}(x-a)^{k} e^{(b-x) t}}{(b-a)^{m} k !}=\sum_{n=0}^{\infty} \mathbb{Y}_{k}^{n}(x ; a, b, m) \frac{t^{n}}{n !},
$$

where $k=0,1, \ldots, n$.

By using (7), we have

$$
\mathbb{Y}_{k}^{n}(x ; a, b, m)=\left(\begin{array}{l}
n \\
k
\end{array}\right) \frac{(x-a)^{k}(b-x)^{n-k}}{(b-a)^{m}}
$$

where

$$
\left(\begin{array}{l}
n \\
k
\end{array}\right)=\frac{n !}{k !(n-k) !}
$$

(cf. [34]; see also [17,26-35]). 
Substituting $a=0$ and $b=1$ into (8), we have the Bernstein basis functions:

$$
B_{k}^{n}(x)=\left(\begin{array}{l}
n \\
k
\end{array}\right) x^{k}(1-x)^{n-k}
$$

(cf. $[8,10,12,18])$.

\subsection{The Family of Beta Distributions}

Many interesting analogs of the beta distribution were investigated by Johnson et al. [3] (Equation (25.1), (p. 210)). A family of beta distributions, which is composed of all distributions with probability density functions, is defined by

$$
P_{Y}(x ; a, b ; p, q)=\frac{1}{B(p, q)} \frac{(x-a)^{p-1}(b-x)^{q-1}}{(b-a)^{p+q-1}}
$$

where $a \leq x \leq b, p \geq 0, q \geq 0$, and

$$
\begin{aligned}
B(p, q) & =\int_{0}^{1} u^{p-1}(1-u)^{q-1} d u \\
& =\frac{\Gamma(p) \Gamma(q)}{\Gamma(p+q)}
\end{aligned}
$$

where $B(p, q)$ denotes the beta function, which is a symmetric function and related to the gamma function $\Gamma(p)$ with

$$
\Gamma(k+1)=k !
$$

for $k \in \mathbb{N}_{0}$ (cf. [3], see also [23,24,31,37,38]).

Substituting $q=1$ into (10), we have the power-function distribution.

For $y=\frac{x-a}{b-a}$, the distribution $P_{Y}(x ; a, b ; p, q)$ reduces to the following well-known beta probability density function $P_{B}(y ; p, q)$ :

$$
P_{B}(y ; p, q)=\frac{1}{B(p, q)} y^{p-1}(1-y)^{q-1}
$$

(cf. [3]).

Probability density function $P_{B}(y ; p, q)$ has reflection symmetry property.

As the beta function $B(p, q)$ is a symmetric function, that is,

$$
B(p, q)=B(q, p),
$$

we have

$$
P_{B}(y ; p, q)=P_{B}(1-y ; q, p) .
$$

The function $P_{B}(y ; p, q)$ is also known as the standard form of the beta distribution with parameters $p$ and $q$.

When $q=1$, the function $P_{B}(y ; p, q)$ reduces to the following standard power-function,

$$
P_{B}(y ; p, 1)=p y^{p-1}
$$

(cf. [3] (p. 210)).

Let us briefly summarize the following sections of this paper.

In Section 2, using generating functions with their functional equations, some identities associated with the Bernstein-type basis functions, the Apostol-Bernoulli numbers, $\lambda$-Stirling numbers, and $\lambda$-array polynomials are given. 
In Section 3, beta type distributions in terms of the Bernstein-type basis functions are defined. Many properties including symmetry property of these distributions are given. Series representations for these distributions are given.

In Section 4, we give moment generating function for beta type distributions. We give many properties of this function. With the aid of the moment generating function, we give moments and expected value for the logarithm of random variable of the beta type distributions. We give some identities and formulas including generalized harmonic numbers, the Lah numbers, the Stirling numbers, and the polygamma function. Finally, we give some numerical values of these expected value for the logarithm of random variable in terms of the polygamma function, the digamma function.

In Section 5, we give our results with our future studies.

\section{Identities Including Bernstein-Type Basis Functions, Apostol-Bernoulli Numbers and Array Type Polynomials}

In this section, using functional equations for generating functions, we give some identities and relations including the Bernstein-type basis functions, the Apostol-Bernoulli numbers, $\lambda$-Stirling numbers, and $\lambda$-array polynomials.

Theorem 1. Let $a$ and $b$ be non-negative real parameters. Let $\lambda \in \mathbb{R}$. Let $n, m \in \mathbb{N}_{0}, k \in\{0,1, \ldots, n\}$ and $x \in[a, b]$. Then, we have

$$
(b-a)^{m}(b-x)^{n-k} \mathbb{Y}_{k}^{n}(x ; a, b, m)=B_{k}^{n}(x ; a, b) \sum_{c=0}^{n}\left(\begin{array}{l}
n \\
c
\end{array}\right) \mathcal{B}_{n-c}^{(k)}(x ; \lambda) S_{k}^{c}(b-x ; \lambda) .
$$

Proof. We set the following functional equation:

$$
f_{\mathbb{Y}, k}(x, t ; a, b, m)=\frac{(x-a)^{k}}{(b-a)^{m}} F_{B}(t, x, k ; \lambda) F_{A}(t,(b-x), k ; \lambda) .
$$

Combining the above functional equation with Equations (1), (2), and (7), we get

$$
\sum_{n=0}^{\infty} \mathbb{Y}_{k}^{n}(x ; a, b, m) \frac{t^{n}}{n !}=\frac{(x-a)^{k}}{(b-a)^{m}} \sum_{n=0}^{\infty} \mathcal{B}_{n}^{(k)}(x ; \lambda) \frac{t^{n}}{n !} \sum_{n=0}^{\infty} S_{k}^{n}(b-x ; \lambda) \frac{t^{n}}{n !}
$$

After doing some algebraic calculations in the above equation, with the help of the Cauchy product rule, we obtain the following equation.

$$
\begin{gathered}
(b-a)^{m}(b-x)^{n-k} \sum_{n=0}^{\infty} \mathbb{Y}_{k}^{n}(x ; a, b, m) \frac{t^{n}}{n !} \\
=B_{k}^{n}(x ; a, b) \sum_{n=0}^{\infty} \sum_{c=0}^{n}\left(\begin{array}{l}
n \\
c
\end{array}\right) \mathcal{B}_{n-c}^{(k)}(\lambda) S_{k}^{c}(b-x ; \lambda) \frac{t^{n}}{n !} .
\end{gathered}
$$

Equating the coefficients of $\frac{t^{n}}{n !}$ on both sides of the previous equation, proof of the theorem is completed.

Theorem 2. Let $a$ and $b$ be non-negative real parameters. Let $\lambda \in \mathbb{R}$. Let $n, m \in \mathbb{N}_{0}, k \in\{0,1, \ldots, n\}$ and $x \in[a, b]$. Then, we have

$$
\mathbb{Y}_{k}^{n}(x ; a, b, m)=\frac{(x-a)^{k}}{(b-a)^{m}} \sum_{c=0}^{n}\left(\begin{array}{l}
n \\
c
\end{array}\right) \mathcal{B}_{n-c}^{(k)}(b-x ; \lambda) S_{2}(c, k ; \lambda) .
$$

Proof. We set the following functional equation,

$$
f_{\mathbb{Y}, k}(x, t ; a, b, m)=\frac{(x-a)^{k}}{(b-a)^{m}} F_{B}(t, b-x, k ; \lambda) F_{S}(t, k ; \lambda) .
$$


Combining the previous equation with (1), (3), and (7), we get

$$
\sum_{n=0}^{\infty} \mathbb{Y}_{k}^{n}(x ; a, b, m) \frac{t^{n}}{n !}=\frac{(x-a)^{k}}{(b-a)^{m}} \sum_{n=0}^{\infty} \mathcal{B}_{n}^{(k)}(b-x ; \lambda) \frac{t^{n}}{n !} \sum_{n=0}^{\infty} S_{2}(n, k ; \lambda) \frac{t^{n}}{n !} .
$$

After doing some algebraic calculations in the above equation, we obtain the following equation.

$$
\sum_{n=0}^{\infty} \mathbb{Y}_{k}^{n}(x ; a, b, m) \frac{t^{n}}{n !}=\frac{(x-a)^{k}}{(b-a)^{m}} \sum_{n=0}^{\infty} \sum_{c=0}^{n}\left(\begin{array}{l}
n \\
c
\end{array}\right) \mathcal{B}_{n-c}^{(k)}(b-x ; \lambda) S_{2}(c, k ; \lambda) \frac{t^{n}}{n !} .
$$

Equating the coefficients of $\frac{t^{n}}{n !}$ on both sides of the previous equation, proof of the theorem is completed.

Substituting $a=0, b=1$ into (11), we get the following corollary.

Corollary 1. Let $\lambda \in \mathbb{R}$. Let $n \in \mathbb{N}_{0}, k \in\{0,1, \ldots, n\}$ and $x \in[0,1]$. Then, we have

$$
B_{k}^{n}(x)=x^{k} \sum_{c=0}^{n}\left(\begin{array}{l}
n \\
c
\end{array}\right) \mathcal{B}_{n-c}^{(k)}(1-x ; \lambda) S_{2}(c, k ; \lambda) \text {. }
$$

\section{Beta Type Distributions}

In this section, we define beta type distributions in terms of the Bernstein-type basis functions. We investigate some properties of these distributions. We also give symmetry property of these distributions. Moreover, using binomial theorem, we give series representations for these distributions. We give some series and integral representations for these distributions.

Combining (8) with (10), we define the following family of beta type distributions,

$$
F(x ; a, b ; n, m, k)=\frac{\mathbb{Y}_{k}^{n}(x ; a, b, m)}{(b-a)^{n-m+1}\left(\begin{array}{l}
n \\
k
\end{array}\right) B(n-k+1, k+1)},
$$

where $a \neq b, b \geq a ; n, m \in \mathbb{N}_{0}, k \in\{0,1, \ldots, n\}$ and $x \in[a, b]$.

Some properties of these distributions are given as follows.

By using Equation (8), we give the following the symmetry property of the Bernstein basis functions of order $n, \mathbb{Y}_{k}^{n}(x ; a, b, m)$,

$$
\mathbb{Y}_{k}^{n}(a+b-x ; a, b, m)=\mathbb{Y}_{n-k}^{n}(x ; a, b, m) .
$$

Combining the above equation with the following symmetry property of the beta function,

$$
B(n-k+1, k+1)=B(k+1, n-k+1),
$$

we obtain symmetry property of the distribution $F(x ; a, b ; n, m, k)$, described as follows,

$$
F(a+b-x ; a, b ; n, m, k)=F(x ; a, b ; n, m, n-k) .
$$

For all $x \in[a, b]$, we have

$$
F(x ; a, b ; n, m, k) \geq 0,
$$

and

$$
\int_{a}^{b} F(x ; a, b ; n, m, k) d x=1
$$


In order to compute the above integration, we need the following well-known integral formula,

$$
\int_{a}^{b}(x-a)^{\alpha-1}(b-x)^{\beta-1} d x=(b-a)^{\alpha+\beta-1} B(\alpha, \beta)
$$

(cf. [24,35,37], (p. 9, Equation (60)).

Substituting (12) into (13), we have

$$
\int_{a}^{b} F(x ; a, b ; n, m, k) d x=\frac{1}{(b-a)^{n-m+1}\left(\begin{array}{l}
n \\
k
\end{array}\right) B(n-k+1, k+1)} \int_{a}^{b} \mathbb{Y}_{k}^{n}(x ; a, b, m) d x .
$$

Combining the above equation with (8), we get

$$
\int_{a}^{b} F(x ; a, b ; n, m, k) d x=\frac{1}{(b-a)^{n+1} B(n-k+1, k+1)} \int_{a}^{b}(x-a)^{k}(b-x)^{n-k} d x .
$$

Combining (15) with (14), after some elementary calculations, we arrive at (13).

Substituting $k=u, n-k=v$ into (12), we have

$$
F_{1}(x ; a, b ; u+v, u)=\frac{(x-a)^{u}(b-x)^{v}}{(b-a)^{u+v+1} B(v+1, u+1)},
$$

where $a \neq b, b \geq a, x \in[a, b], u, v \in(0, \infty)$.

Consequently, we see that Equation (16) is modified of the Equation (10).

Remark 1. Observe that when $a=0, b=1$, we have

$$
F(x ; 0,1 ; n, m, k)=\frac{\mathbb{Y}_{k}^{n}(x ; 0,1, m)}{\left(\begin{array}{l}
n \\
k
\end{array}\right) B(n-k+1, k+1)} .
$$

Combining the above equation with Equation (9), for $0 \leq x \leq 1$ and $k=0,1,2, \ldots, n$, we have the following well-known binomial (Newton) distribution,

$$
(n+1)\left(\begin{array}{l}
n \\
k
\end{array}\right) B(n-k+1, k+1) F(x ; 0,1 ; n, n, k)=B_{k}^{n}(x),
$$

(cf. $[3,19,26,27,31,34])$.

Remark 2. By using discrete random variable and the Bernstein basis functions of order $n$, binomial distribution and moment generating functions have been studied ([18-20,26,27,34]). By modifying Equation (7), Simsek [26] gave the following moment generating functions $M_{x}(t, x: n ; a, b)$ related to the following Bernstein basis functions of order $n$,

$$
B_{k}^{n}(x ; a, b)=\left(\begin{array}{l}
n \\
k
\end{array}\right)\left(\frac{x-a}{b-a}\right)^{k}\left(\frac{b-x}{b-a}\right)^{n-k}
$$

where $k=0,1, \cdots, n$ and $x \in[a, b]$ and also $0 \leq\left|\frac{x-a}{b-a}\right| \leq 1,0 \leq\left|\frac{b-x}{b-a}\right| \leq 1$, that is, $M_{x}(t, x: n ; a, b)$ is given by

$$
M_{x}(t, x: n ; a, b)=\left(e^{t} \frac{x-a}{b-a}+\frac{b-x}{b-a}\right)^{n} .
$$

Substituting $a=0$ and $b=1$ into the above function, we have the following well-known formul for the moment generating function of the Binomial distribution:

$$
M_{x}(t, x: n ; 0,1)=\left(e^{t} x+1-x\right)^{n}
$$




\section{(cf. $[18-20,26,27,34])$.}

\section{Series Representations for Beta Type Distributions}

Here, by using binomial theorem, we give some series representations for the distribution $F(x ; a, b ; n, m, k)$.

Theorem 3. We assume that $\left|\frac{b-x}{b-a}\right|<1$. Then, we have

$$
\sum_{n=k}^{\infty} \frac{F(x ; a, b ; n, m, k)}{n+1}=\frac{1}{x-a}
$$

where $x \neq a$.

Proof. In [13] (Equation (1.9)), by using binomial theorem, for $|x|<1$, Gould gave the following series,

$$
\sum_{n=k}^{\infty}\left(\begin{array}{l}
n \\
k
\end{array}\right) x^{n-k}=\frac{1}{(1-x)^{k+1}}
$$

Combining (12) with (18) and (8), we get

$$
\sum_{n=k}^{\infty} \frac{F(x ; a, b ; n, m, k)}{n+1}=\frac{(x-a)^{k}}{(b-a)^{k+1}} \sum_{n=k}^{\infty}\left(\frac{b-x}{b-a}\right)^{n-k} .
$$

Observe that Equation (17) is modified as follows,

$$
\sum_{n=0}^{\infty} \frac{F(x ; a, b ; n+k, m, k)}{n+k+1}=\frac{1}{x-a} .
$$

Theorem 4. We assume that $\left|\frac{b-x}{b-a}\right|<1$. Then, we have

$$
\sum_{n=0}^{\infty} \frac{F(x ; a, b ; n, m, k)}{\left(\begin{array}{l}
n \\
k
\end{array}\right)}=(b-a) \frac{(x-a)^{k-2}}{(b-x)^{k}}
$$

where $x \neq a$ and $x \neq b$.

Proof. By using (8) and (12), we obtain

$$
\sum_{n=0}^{\infty} \frac{F(x ; a, b ; n, m, k)}{\left(\begin{array}{c}
n \\
k
\end{array}\right)}=\frac{\left(\frac{x-a}{b-a}\right)^{k}}{(b-a)} \sum_{n=0}^{\infty}(n+1)\left(\frac{b-x}{b-a}\right)^{n} .
$$

After some elementary calculations in the above equation, we arrive at the desired result.

\section{Moment Generating Function for Beta Type Distributions}

In this section, we give moment generating function for beta type distributions. We investigate some properties of this function. By using these functions, we find moments and expected value for the logarithm of random variable of the beta type distributions. We give relations among generalized harmonic number, the Lah numbers, the Stirling numbers, and the polygamma function. Finally, we give some numarical values of these expected value for the logarithm of random variable in terms of the polygamma function, the digamma function. 
Moment generating function for the distribution $F(x ; a, b ; n, m, k)$ is given as follows,

$$
\begin{aligned}
& M_{x}(t ; a, b ; n, m, k) \\
& =\sum_{j=0}^{\infty}\left(\sum_{c=0}^{k}(-1)^{k-c}\left(\begin{array}{l}
k \\
c
\end{array}\right) \frac{B(c+j+1, n-k+1)-a^{k-c} b^{c+j+n-k+1}}{(b-a)^{n+1} B(n-k+1, k+1)}\right) \frac{t^{j}}{j !} .
\end{aligned}
$$

Combining the above equation with (14), we obtain the following moment generating function for $F(x ; a, b ; n, m, k)$ :

$$
M_{x}(t ; a, b ; n, m, k)=\sum_{j=0}^{\infty}\left(\sum_{c=0}^{k}(-1)^{k-c}\left(\begin{array}{c}
k \\
c
\end{array}\right) \frac{B(c+j+1, n-k+1)-a^{k-c} b^{c+j+n-k+1}}{(b-a)^{n+1} B(n-k+1, k+1)}\right) \frac{t^{j}}{j !} .
$$

By using the moment generating function $M_{x}(t ; a, b ; n, m, k)$, computation formula for the moments is given as follows,

$$
\mu_{l}(a, b ; n, m, k)=\mathbb{E}\left(X^{l}\right)
$$

where $\mathbb{E}(X)$ denotes the expected value or mean of the random variable $X$. That is,

$$
\begin{array}{r}
\mu_{1}(a, b ; n, m, k)=\sum_{c=0}^{k}(-1)^{k-c}\left(\begin{array}{c}
k \\
c
\end{array}\right) a^{k-c} b^{l+c+n-k+1}(b \\
-a)^{-n-1} \frac{B(c+l+1, n-k+1)}{B(n-k+1, k+1)}
\end{array}
$$

or

$$
\mu_{l}(a, b ; n, m, k)=\left.\frac{\partial^{l}}{\partial t^{l}}\left\{M_{x}(t ; a, b ; n, m, k)\right\}\right|_{t=0} .
$$

Replacing $X$ by $e^{-t \ln (x-a)}$ in the above equation, we arrive at the following theorem.

Theorem 5. Let $n, m \in \mathbb{N}_{0}, k \in\{0,1, \ldots, n\}$ and $x \in[a, b]$. Then, we have

$$
\begin{aligned}
& \mathbb{E}\left(e^{-t \ln (x-a)}\right) \\
& =\sum_{v=0}^{\infty} \sum_{j=0}^{v} \sum_{l=0}^{v}(-1)^{v-l+j}\left(\begin{array}{c}
v \\
l
\end{array}\right) \frac{(b-a)^{m+l} s_{1}(v, j) B(k+l+1, n-k+1)}{v ! B(n-k+1, k+1)} t^{j}
\end{aligned}
$$

where $x-a>0$ and $b \neq a$.

Proof. By using definition of the expected value $\mathbb{E}(X)$, we have

$$
\begin{aligned}
& \mathbb{E}\left(e^{-t \ln (x-a)}\right) \\
= & \sum_{j=0}^{\infty}(-1)^{j} \frac{t^{j}}{j !} \int_{a}^{b} \frac{(x-a)^{k}(b-x)^{n-k}}{(b-a)^{n-m+1} B(n-k+1, k+1)}(\ln (x-a))^{j} d x .
\end{aligned}
$$

Combining the above equation with (5), we obtain

$$
\begin{aligned}
& \mathbb{E}\left(e^{-t \ln (x-a)}\right) \\
& =\sum_{j=0}^{\infty}(-1)^{j}\left(\begin{array}{c}
v \\
l
\end{array}\right) t^{j} \sum_{v=0}^{\infty} \frac{S_{1}(v, j)}{v !(b-a)^{n-m+1}} \int_{a}^{b} \frac{(x-a-1)^{v}(x-a)^{k}(b-x)^{n-k}}{B(n-k+1, k+1)} d x .
\end{aligned}
$$


Therefore,

$$
\mathbb{E}\left(e^{-t \ln (x-a)}\right)=\sum_{j=0}^{\infty} \sum_{v=0}^{\infty} \frac{(-1)^{j} t^{j} S_{1}(v, j)}{v ! B(n-k+1, k+1)(b-a)^{n-m+1}} \sum_{l=0}^{v}(-1)^{v-l}\left(\begin{array}{c}
v \\
l
\end{array}\right)
$$

As $S_{1}(v, j)=0$ for $j>v$, combining the above equation with (14), we arrive at the desired result.

Theorem 6. Let $n, m \in \mathbb{N}_{0}, k \in\{0,1, \ldots, n\}$. Then, we have

$$
\begin{aligned}
& \sum_{d=0}^{\infty} \sum_{k=0}^{d}\left(\begin{array}{l}
d \\
k
\end{array}\right) \frac{(-a-1)^{d-k} \mu_{k}}{d !}(-t)_{(d)} \\
& =\sum_{v=0}^{\infty} \sum_{j=0}^{v} \sum_{l=0}^{v}(-1)^{v-l+j}\left(\begin{array}{c}
v \\
l
\end{array}\right) \frac{(b-a)^{m+l} S_{1}(v, j) B(k+l+1, n-k+1)}{v ! B(n-k+1, k+1)} t^{j}
\end{aligned}
$$

where $b \neq a$ and

$$
\mu_{d}=\mathbb{E}\left(x^{d} ; a, b\right) .
$$

Proof. By using (19), we obtain

$$
\begin{aligned}
& \sum_{m=0}^{\infty} \mathbb{E}\left(\frac{(\ln (x-a))^{m}}{m !}\right) t^{m} \\
= & \sum_{v=0}^{\infty} \sum_{j=0}^{v} \sum_{l=0}^{v}(-1)^{v-l+j}\left(\begin{array}{l}
v \\
l
\end{array}\right) \frac{(b-a)^{m+v-l-1} S_{1}(v, j) B(k+v-l+1, n-k+1)}{v ! B(n-k+1, k+1)} t^{j} .
\end{aligned}
$$

By combining the previous equation with (5), we get

$$
\begin{aligned}
& \mathbb{E}\left(\sum_{c=0}^{\infty} \sum_{d=0}^{\infty} S_{1}(d, c) \frac{(x-a-1)^{d}}{d !}(-t)^{c}\right) \\
& =\sum_{v=0}^{\infty} \sum_{j=0}^{v} \sum_{l=0}^{v}(-1)^{v-l+j}\left(\begin{array}{c}
v \\
l
\end{array}\right) \frac{(b-a)^{m+l} S_{1}(v, j) B(k+l+1, n-k+1)}{v ! B(n-k+1, k+1)} t^{j}
\end{aligned}
$$

Therefore,

$$
\begin{aligned}
& \sum_{d=0}^{\infty} \sum_{c=0}^{d} \sum_{k=0}^{d}\left(\begin{array}{l}
d \\
k
\end{array}\right) S_{1}(d, c) \frac{(-a-1)^{d-k} \mathbb{E}\left(x^{k}\right)}{d !}(-t)^{c} \\
& =\sum_{v=0}^{\infty} \sum_{j=0}^{v} \sum_{l=0}^{v}(-1)^{v-l+j}\left(\begin{array}{l}
v \\
l
\end{array}\right) \frac{(b-a)^{m+l} S_{1}(v, j) B(k+l+1, n-k+1)}{v ! B(n-k+1, k+1)} t^{j}
\end{aligned}
$$

Combining the above equation with Equation (6), after some elementary calculation, we arrive at the desired result.

We give some special values of (21) as follows,

$$
\begin{gathered}
B(n+1-k, k+1)\left(\beta_{0} P_{0}(-t)+\beta_{1} P_{1}(-t)+\cdots+\beta_{d} P_{d}(-t)+\cdots\right)= \\
\sum_{v=0}^{\infty} \frac{(-1)^{v}(b-a)^{v+m-1}}{v !}\left(S_{1}(v, 0)-S_{1}(v, 1) t+\cdots+(-1)^{v} S_{1}(v, v) t^{v}\right) \\
\left(B(v+k+1, n+1-k)+\ldots+(-1)^{v} \frac{B(k+1, n+1-k)}{(b-a)^{v}}\right.
\end{gathered}
$$

where

$$
\beta_{d}=\sum_{k=0}^{d}\left(\begin{array}{l}
d \\
k
\end{array}\right) \frac{(-a-1)^{d-k} \mu_{k}}{d !}
$$


and

$$
P_{d}(t)=t(t-1) \ldots(t-d+1)=\sum_{m=0}^{d} S_{1}(d, m) t^{m} .
$$

After some elementary calculations in the above equation, we have

$$
\begin{aligned}
P_{d}(t)= & \delta_{d, 0}+(-1)^{d-1}(d-1) ! t+(-1)^{d-2}(d-1) ! \frac{H_{d-1}}{1 !} t^{2} \\
& +(-1)^{d-3}(d-1) ! \frac{\left(H_{d-1}\right)^{2}-H_{d-1}^{(2)}}{2 !} t^{3} \\
& +(-1)^{d-4}(d-1) ! \frac{\left(H_{d-1}\right)^{3}-3 H_{d-1} H_{d-1}^{(2)}+2 H_{d-1}^{(3)}}{3 !} t^{4} \\
& +\cdots-\frac{d(d-1)}{2} t^{d-1}+t^{d}
\end{aligned}
$$

where $\delta_{d, 0}$ denotes the Kronecker delta,

$$
\delta_{d, 0}= \begin{cases}1 & \text { if } d=0 \\ 0 & \text { if } d \neq 0\end{cases}
$$

and $H_{d}$ denotes the harmonic numbers and $H_{d}^{(j)}$ denotes the generalized harmonic number of order $j$ of $d$, which are defined as follows,

$$
H_{d}^{(j)}=\sum_{c=1}^{d} \frac{1}{c^{j}}
$$

and $H_{d}=H_{d}^{(1)}$ (see, for details, $[9,25,38]$ ).

We modify the Equation (21) as follows.

$$
\begin{aligned}
& \sum_{d=0}^{\infty} \sum_{k=0}^{d}\left(\begin{array}{l}
d \\
k
\end{array}\right) \frac{(-a-1)^{d-k} \mu_{k}}{d !}(-t)_{(d)} \\
& =\sum_{v=0}^{\infty} \sum_{l=0}^{v}(-1)^{v-l}\left(\begin{array}{c}
v \\
l
\end{array}\right) \frac{(b-a)^{m+l} B(k+l+1, n-k+1)}{v ! B(n-k+1, k+1)} \sum_{j=0}^{v} S_{1}(v, j)(-t)^{j}
\end{aligned}
$$

where

$$
P_{v}(-t)=\sum_{j=0}^{v} S_{1}(v, j)(-t)^{j}=(-t)_{(v)}=\sum_{j=0}^{v} L(v, j) t_{(j)}
$$

where $L(v, j)$ denotes the Lah numbers. Consequently, we get the following corollaries.

Corollary 2. Let $n, m \in \mathbb{N}_{0}, k \in\{0,1, \ldots, n\}$. Then, we have

$$
\sum_{d=0}^{\infty} \beta_{d} P_{d}(-t)=\sum_{v=0}^{\infty}(-t)_{(v)} \sum_{l=0}^{v}(-1)^{v-l}\left(\begin{array}{l}
v \\
l
\end{array}\right) \frac{(k+1) !(n+1) !(b-a)^{m+l}}{k !(n+l+1) !}
$$

where $b \neq a$.

Corollary 3. Let $n, m \in \mathbb{N}_{0}, k \in\{0,1, \ldots, n\}$. Then, we have

$$
\begin{aligned}
& \sum_{d=0}^{\infty} \beta_{d} P_{d}(-t) \\
& =\sum_{v=0}^{\infty} \sum_{j=0}^{v} L(v, j) t_{(j)} \sum_{l=0}^{v}(-1)^{v-l}\left(\begin{array}{c}
v \\
l
\end{array}\right) \frac{(k+1) !(n+1) !(b-a)^{m+l}}{k !(n+l+1) !}
\end{aligned}
$$


where $b \neq a$.

Corollary 4. Let $n, m \in \mathbb{N}_{0}, k \in\{0,1, \ldots, n\}$. Then, we have

$$
\sum_{d=0}^{\infty} \beta_{d} P_{d}(-t)=\sum_{v=0}^{\infty} \sum_{l=0}^{v}(-1)^{v-l}\left(\begin{array}{l}
v \\
l
\end{array}\right) \frac{(k+1) !(n+1) !(b-a)^{m+l}}{k !(n+l+1) !} P_{v}(-t)
$$

where $b \neq a$.

\section{Expected Value for the Logarithm of Random Variable of Beta Type Distributions}

Here, we give some identities and relations associated with the polygamma functions, the digamma function, and the expected value for the logarithm of random variable of the beta type distributions.

Theorem 7. Let $j \in \mathbb{N}_{0}$. Then, we have

$$
\begin{aligned}
& \mathbb{E}\left((\ln (x-a))^{j}\right) \\
= & \frac{1}{B(v+1, u+1)} \sum_{m=0}^{j}\left(\begin{array}{c}
j \\
m
\end{array}\right) \ln ^{m}(b-a) \frac{\partial^{j-m}}{\partial u^{j-m}}\{B(u+1, v+1)\},
\end{aligned}
$$

where $x-a>0$ and $b \neq a$.

Proof. By using (20) and (16), we have

$$
\mathbb{E}\left((\ln (x-a))^{j}\right)=\int_{a}^{b} F_{1}(x ; a, b ; u+v, u) \ln ^{j}(x-a) d x,
$$

where $x-a>0$ and $b \neq a$. Thus, we get

$$
\begin{aligned}
& \mathbb{E}\left((\ln (x-a))^{j}\right) \\
= & \frac{1}{(b-a)^{u+v+1} B(v+1, u+1)} \int_{a}^{b}(x-a)^{u}(b-x)^{v} \ln ^{j}(x-a) d x \\
= & \frac{1}{(b-a)^{u+v+1} B(v+1, u+1)} \frac{\partial^{j}}{\partial u^{j}}\left\{\int_{a}^{b}(x-a)^{u}(b-x)^{v} d x\right\},
\end{aligned}
$$

where $x \neq a$ and $b \neq a$. Combining the above equation with (14), we get

$$
\begin{aligned}
& \mathbb{E}\left((\ln (x-a))^{j}\right) \\
= & \frac{1}{(b-a)^{u+v+1} B(v+1, u+1)} \frac{\partial^{j}}{\partial u^{j}}\left\{(b-a)^{u+v+1} B(u+1, v+1)\right\} .
\end{aligned}
$$

By applying the Leibniz formula for differentiation, with respect to $u$, to the following function

$$
(b-a)^{u+v+1} B(u+1, v+1),
$$

after some elementary calculations, we arrive at the desired result.

Note that by using mathematical induction technique, proof of (22) is given.

Finally, we give few values of the expected value for the logarithm of random variable of the beta type distributions by applying the formula (22) (with $j=1,2,3,4$ ) as follows. 
For $j=1$, we have

$$
\mathbb{E}(\ln (x-a))=\ln (b-a)+\psi(u+1)-\psi(u+v+2)
$$

It is well known that the logarithm of the geometric mean of a distribution (with random variable $X)$ is the arithmetic mean of $\ln (X)$, equivalently, its expected value is $\mathbb{E}(\ln (X))$.

For the beta distribution, the expected value with the help of integral, is given as follows.

$$
\mathbb{E}(\ln (x))=\psi(u+1)-\psi(u+v+2)
$$

(cf. [3]).

For $j=2$, we have

$$
\begin{aligned}
\mathbb{E}\left((\ln (x-a))^{2}\right)= & \ln ^{2}(b-a)+2 \ln (b-a)(\psi(u+1)-\psi(u+v+2)) \\
& +(\psi(u+1)-\psi(u+v+2))^{2}+\left(\psi^{(1)}(u+1)-\psi^{(1)}(u+v+2)\right) .
\end{aligned}
$$

For $j=3$, we have

$$
\begin{aligned}
\mathbb{E}\left((\ln (x-a))^{3}\right)= & \ln ^{3}(b-a)+3\left(\psi^{(1)}(u+1)-\psi^{(1)}(u+v+2)\right) \\
& \times(\psi(u+1)-\psi(u+v+2)) \\
& +(\psi(u+1)-\psi(u+v+2))^{3}+\left(\psi^{(2)}(u+1)-\psi^{(2)}(u+v+2)\right) \\
& +3 \ln ^{2}(b-a)(\psi(u+1)-\psi(u+v+2)) \\
& +3 \ln (b-a)\left((\psi(u+1)-\psi(u+v+2))^{2}\right) \\
& +3 \ln (b-a)\left(\psi^{(1)}(u+1)-\psi^{(1)}(u+v+2)\right),
\end{aligned}
$$

and for $j=4$, we have

$$
\begin{aligned}
\mathbb{E}\left((\ln (x-a))^{4}\right)= & \ln ^{4}(b-a)+(\psi(u+1)-\psi(u+v+2))^{4} \\
& +6\left(\psi^{(1)}(u+1)-\psi^{(1)}(u+v+2)\right)(\psi(u+1)-\psi(u+v+2))^{2} \\
& +4\left(\psi^{(2)}(u+1)-\psi^{(2)}(u+v+2)\right)(\psi(u+1)-\psi(u+v+2)) \\
& +3\left(\psi^{(1)}(u+1)-\psi^{(1)}(u+v+2)\right)^{2} \\
& +\left(\psi^{(3)}(u+1)-\psi^{(3)}(u+v+2)\right) \\
& +4 \ln ^{3}(b-a)(\psi(u+1)-\psi(u+v+2)) \\
& +6 \ln ^{2}(b-a)(\psi(u+1)-\psi(u+v+2))^{2} \\
& +6 \ln ^{2}(b-a)\left(\psi^{(1)}(u+1)-\psi^{(1)}(u+v+2)\right) \\
& +4 \ln ^{(}(b-a)(\psi(u+1)-\psi(u+v+2))^{3} \\
& +12 \ln (b-a)\left(\psi^{(1)}(u+1)-\psi^{(1)}(u+v+2)\right) \\
& \times(\psi(u+1)-\psi(u+v+2)) \\
& +4 \ln (b-a)\left(\left(\psi^{(2)}(u+1)-\psi^{(2)}(u+v+2)\right)\right),
\end{aligned}
$$

and so on.

\section{Conclusions}

In this paper, using the Bernstein-type basis functions of order $n$, we defined a new class of beta type distributions. We gave not only symmetry property and other properties of these distributions, but, using the Bernstein-type basis functions and binomial series, we also gave series and integral 
representations of these distributions. We defined moment generating function and expected values for the logarithm of random variable of this distribution. By using this generating function, we gave moments related to the Harmonic numbers; the Stirling numbers; the Lah numbers; and we give many new relations and identities among this distribution, expected values, the polygamma function, the digamma function, the harmonic numbers, the Stirling numbers, the polygamma function, and the digamma function. By using functional equations of generating functions, we gave some relations and identities including the Bernstein-type basis functions, the $\lambda$-array polynomials, and the Apostol-Bernoulli numbers and polynomials. Some numerical values of the expected values were given. The logarithm of the geometric mean of a distribution with random variable is the arithmetic mean of $\mathbb{E}(\ln (X))$, which has many applications in mathematical models, in quantities of information (entropy), in Bayesian inference, in probability integral transform, in distribution of any individual order statistic, and in other disciplines. The results of this paper may potentially be used in mathematics, in physics, in engineering, in health sciences, and also in social sciences.

We plan to investigate connection with the Bernstein-type basis functions of order $n$, moments in terms of moment generating function for beta type distributions, the Hankel determinant, the Hilbert matrix, and orthogonal polynomials, in near future.

Author Contributions: The authors are equally contribute the content of the paper. All authors have read and agreed to the published version of the manuscript.

Funding: This research received no external funding.

Acknowledgments: The present investigation was supported by Scientific Research Project Administration of Akdeniz University (Project ID: FBA-2020-5299).

Conflicts of Interest: The authors declare no conflicts of interest. 


\section{References}

1. Bagarello, F.; Gargano, F.; Spagnolo, S.; Triolo, S. Coordinate representation for non-Hermitian position and momentum operators. Proc. R. Soc. A Math. Phys. Eng. Sci. 2017, 473, 1-13. [CrossRef]

2. Gupta, A.K.; Nadarajah, S. Handbook of Beta Distribution and Its Applications; 1st ed.; CRC Press: Boca Raton, FL, USA, 2004; ISBN9780429152887

3. Johnson, N.L.; Kotz, S.; Balakrishnan, N. Continuous Univariate Distributions; 2nd ed.; Wiley-Interscience Publication John Wiley \& Sons, Inc.: New York, NY, USA; Chichester, UK; Brisbane, Australia; Toronto, Italy; Singapore, 1995; Volume 2.

4. Oguamanam, D.C.D.; Martin, H.R.; Huissoon, J.P. On the application of the beta Distribution to gear damage analysis. Appl. Acoust. 1993, 45, 247-261. [CrossRef]

5. Trapani, C.; Triolo, S.; Tschinkei F. Distribution frames and bases. J. Fourier Anal. Appl. 2019, 25, 2109 -2140. [CrossRef]

6. Acikgoz, M.; Araci, S. On generating function of the Bernstein polynomials. Numerical Anal. Appl. Math. Amer. Inst. Phys. Conf. Proc. 2010, CP1281, 1141-1143.

7. Bayad, A.; Kim, T.; Lee, S.H.; Dolgy, D.V. A note on the generalized Bernstein polynomials. Honam Math. J. 2011, 33, 1-11. [CrossRef]

8. Bernstein, S.N. Démonstration du théorème de Weierstrass fondée sur la calcul des probabilités. Comm. Soc. Math. Charkow Sér. 1912, 13, 1-2.

9. Graham, R.L.; Knuth, D.E.; Patashnik, O. Concrete Mathematics; Addison-Wesley: Boston, MA, USA, 1994.

10. Farouki, R.T. Pythagorean-Hodograph Curves: Algebra and Geometry Inseparable; Springer: Berlin/Heidelberg, Germany; New York, NY, USA, 2008.

11. Farouki, R.T. The Bernstein polynomial basis: A centennial retrospective. Comput Aided Geom. Des. 2012, 29, 379-419. [CrossRef]

12. Goldman, R. Identities for the Univariate and Bivariate Bernstein Basis Functions; Paeth, A.; Ed.; Graphics Gems V; Academic Press: Cambridge, MA, USA, 1995; pp. 149-162.

13. Gould, H.W. Fundamentals of Series: Table II: Examples of Series Which Appear in Calculus. Quaintance J. Ed. 2010. Available online: https://math.wvu.edu/ hgould/Vol.2.PDF (accessd on 14 April 2020)

14. Khidr, A.M.; El-Desouky, B.S. A Symmetric Sum Involving the Stirling Numbers of the First Kind. Eur. J. Comb. 1984, 5, 51-54. [CrossRef]

15. Kim, M.S.; Kim, D.; Kim, T. On the $q$-Euler numbers related to modified $q$-Bernstein polynomials. Abstr. Appl. Anal. 2010, 2010, 1-15.

16. Kim, W.J.; Kim, D.S.; Kim, H.Y.; Kim, T. Some identities of degenerate Euler polynomials associated with degenerate Bernstein polynomials. J. Inequal. Appl. 2019, 2019. [CrossRef]

17. Kucukoglu, I.; Simsek, Y. A note on generating functions for the unification of the Bernstein-type basis functions. Filomat 2016, 30, 985-992. [CrossRef]

18. Li, M.; Goldman, R. Note limits of sums for binomial and Eulerian numbers and their associated distributions. Discrete Math. 2020, 343, 111870. [CrossRef] [PubMed]

19. Lorentz, G.G. Bernstein Polynomials; Chelsea Publishing Company: New York, NY, USA, 1986.

20. Lukacs, E. Characteristic Function; 2nd ed.; Charles Griffin \& Company Limited: London, UK, 1970.

21. Luo, Q.M.; Srivastava, H.M. Some generalizations of the Apostol-Genocchi polynomials and the Stirling numbers of the second kind. Appl. Math. Comput. 2011, 217, 5702-5728. [CrossRef]

22. Ozden, H.; Simsek, Y.; Srivastava, H.M. A unified presentation of the generating functions of the generalized Bernoulli, Euler and Genocchi polynomials. Comput. Math. Appl. 2010, 60, 2779-2787. [CrossRef]

23. Rahman, G.; Mubeen, S.; Rehman, A.; Naz, M. On k-Gamma and k-Beta Distributions and Moment Generating Functions. J. Probab. Stat. 2014, 982013, 1-6. [CrossRef]

24. Rainville, E.D. Special Functions; The Macmillan Company: New York, NY, USA, 1960.

25. Riordan, J. Introduction to Combinatorial Analysis; Princeton University Press: Princeton, NJ, USA, 1958.

26. Simsek, B. Formulas derived from moment generating functions and Bernstein polynomials. Appl. Anal. Discret. Math. 2019, 13, 839-848. [CrossRef]

27. Simsek, B.; Simsek, B. The computation of expected values and moments of special polynomials via characteristic and generating functions. AIP Conf. Proc. 2017, 1863, 300012. [CrossRef] 
28. Simsek, Y. Interpolation Function of Generalized q-Bernstein Type Polynomials and Their Application; Lecture Notes in Computer Science; Springer: Berlin, Germany, 2011; Volume 6920, pp. 647-662.

29. Simsek, Y. On $q$-deformed Stirling numbers. Int. J. Math. Comput. 2012, 15, 1-11.

30. Simsek, Y. Construction a new generating function of Bernstein-type polynomials. Appl. Math. Comput. 2011, 218, 1072-1076. [CrossRef]

31. Simsek, Y. q-Beta Polynomials and their Applications. Appl. Math. Inf. Sci. 2013, 7, 2539-2547. [CrossRef]

32. Simsek, Y. Functional equations from generating functions: A novel approach to deriving identities for the Bernstein basis functions. Fixed Point Theory Appl. 2013, 2013, 1-13. [CrossRef]

33. Simsek, Y. Generating functions for generalized Stirling type numbers, Array type polynomials, Eulerian type polynomials and their applications. Fixed Point Theory Appl. 2013, 2013, 1-28. [CrossRef]

34. Simsek, Y. Generating functions for the Bernstein type polynomials: A new approach to deriving identities and applications for the polynomials. Hacet. J. Math. Stat. 2014, 43, 1-14.

35. Simsek, Y. Analysis of the Bernstein basis functions: An approach to combinatorial sums involving binomial coefficients and Catalan numbers. Math. Meth. Appl. Sci. 2015, 38, 3007-3021. [CrossRef]

36. Simsek, Y. Explicit formulasfor $p$-adic integrals: Approach to $p$-adic distributions and some families of special numbers and polynomials. Montes Taurus J. Pure Appl. Math. 2019, 1, 1-76.

37. Srivastava, H.M.; Choi, J. Series Associated with the Zeta and Related Functions; Kluwer Acedemic Publishers: Dordrecht, The Netherlands; Boston, MA, USA; London, UK, 2001.

38. Srivastava, H.M.; Choi, J. Zeta and q-Zeta Functions and Associated Series and Integrals; Elsevier Science Publishers: Amsterdam, The Netherlands; London, UK; New York, NY, USA, 2012.

(C) 2020 by the authors. Licensee MDPI, Basel, Switzerland. This article is an open access article distributed under the terms and conditions of the Creative Commons Attribution (CC BY) license (http:/ / creativecommons.org/licenses/by/4.0/). 